\title{
Asociación entre embarazo adolescente e hiperbilirrubinemia y bajo peso al nacer: cohorte retrospectiva en un hospital en Perú, 2015-2016 Association between adolescent pregnancy and hyperbilirubinemia and low weight at birth: retrospective cohorte in a hospital in Perú, 2015-2016
}

\author{
Cindy Hernández Maldonado ${ }^{1, a}$, Jaime Rosales Rimache $e^{2, b}$ \\ ${ }^{1}$ Facultad de Medicina Humana y Ciencias de la Salud, Universidad Alas Peruanas. Lima, Perú. \\ ${ }^{2}$ Facultad de Salud Pública y Administración, Universidad Peruana Cayetano Heredia. Lima, Perú. \\ ${ }^{a}$ Bachiller en tecnologia médica \\ ${ }^{\mathrm{b}}$ Maestro en salud ocupacional, ORCID: http://orcid.org/0000-0002-1665-2332
}

\section{An Fac med. 2019;80(2):150-6 / DOI: https://10.15381/anales.802.16407}

\section{Correspondencia: \\ Jaime Alonso Rosales Rimache \\ jaime.rosales@upch.pe}

Recibido: 21 de febrero 2019

Aceptado: 22 de abril 2019

Publicación en línea: 28 de junio 2019

Conflictos de interés: Los autores declaran no tener conflictos de interés

Fuente de financiamiento: Autofinanciado

Artículo realizado en base a la tesis presentada por Cindy Hernández Maldonado para obtener el grado académico de Licenciado en Tecnología Médica en el Área de Laboratorio Clínico y Anatomía Patológica

Citar como: Hernández C, Rosales J. Asociación entre embarazo adolescente e hiperbilirrubinemia y bajo peso al nacer: cohorte retrospectiva en un hospital en Perú, 2015-2016. An Fac med. 2019; 80(2):150-6. DOI: https://10.15381/anales.802.16407

\section{Resumen}

Introducción. El embarazo adolescente en Perú es un problema de salud pública que presenta alta prevalencia en regiones como Ica, y se considera como una causa de ciertos desenlaces adversos a la salud del recién nacido como bajo peso e hiperbilirrubinemia. Métodos. Se diseñó una cohorte retrospectiva constituida por gestantes adolescentes entre 13 y 19 años, las cuales fueron divididas en dos grupos: grupo 1 (<18 años de edad) y grupo 2 ( $\geq 18$ años de edad). Resultados. Se evaluaron 481 gestantes adolescentes, de las cuales el grupo 1 representó el $65,3 \%$. Se encontró recién nacidos con bajo peso e hiperbilirrubinemia en el 3,3\% y 18.9\%, respectivamente. El análisis bivariado evidenció diferencias significativas $(p<0,001)$ entre la mediana de edad gestacional entre madres con recién nacidos con peso normal y bajo peso, así como en relación al tipo de parto (cesárea y normal). En cuanto a la hiperbilirrubinemia, tanto el análisis bivariado como el multivariado no evidenciaron diferencias significativas. La regresión de Cox permitió evidenciar que las gestantes adolescentes del grupo 1 tuvieron casi 8 veces más riesgo ( $H R: 7,95, p<0,05)$ de tener un recién nacido con bajo peso en comparación a las gestantes del grupo 2. Conclusión. El embarazo adolescente está asociado al bajo peso al nacer, más no a la hiperbilirrubinemia neonatal.

Palabras clave: Embarazo; Adolescente; Peso al Nacer; Hiperbilirrubinemia

\section{Abstract}

Introduction. Adolescent pregnancy in Peru is a public health problem that has high prevalence in regions such as Ica, and is considered as a cause of certain adverse outcomes to the health of the newborn as low weight and hyperbilirubinemia. Methods. A retrospective cohort was designed consisting of adolescent pregnant women between 13 and 19 years of age, which were divided into two groups: group $1 \quad<18$ years of age) and group 2 ( $\geq 18$ years of age). Results. A total of 481 adolescent pregnant women were evaluated, of which group 1 represented $65,3 \%$. Newborns with low weight and hyperbilirubinemia were found in $3,3 \%$ and $18,9 \%$, respectively. The bivariate analysis showed significant differences $(p<0,001)$ between the median gestational age among mothers with newborns with normal weight and low weight, as well as in relation to the type of delivery (cesarean and normal). Regarding hyperbilirubinemia, both bivariate and multivariate analyzes showed no significant differences. The Cox regression showed that adolescent pregnant women in group 1 had almost 8 times more risk (HR: 7,95, $p<0,05$ ) of having a newborn with low weight compared to the pregnant women in group 2. Conclusion. Adolescent pregnancy is associated with low birth weight, but not with neonatal hyperbilirubinemia.

Keywords: Pregnancy; Adolescent; Birth Weight; Hyperbilirubinemia 


\section{INTRODUCCIÓN}

El embarazo adolescente (EA) es un problema de salud pública en Perú; así, existe un creciente número de casos presentados en los últimos 5 años, donde se ha evidenciado que el 10,5\% de adolescentes son madres y que el 3,5\% están embarazadas de su primer hijo. Esto a consecuencia de los cambios en la conducta sexual, cuyos resultados muestran que el porcentaje de mujeres adolescentes que inició sus relaciones sexuales antes de los 15 años tuvo un incremento en la década $2000-2010$, en especial a partir del año 2009 (de 5,1\% en el 2000 pasó a 6,2\% en el 2009) ${ }^{1}$. Esta tendencia se mantuvo hasta el año 2015, donde la tasa de EA alcanzó un pico máximo de $35,1 \%$ en mujeres menores a los 19 años y en las menores de 15 años de 5,5\% ${ }^{2}$. De acuerdo a la última publicación de la Encuesta Demográfica y de Salud Familiar del año 2017, se evidenció un aumento proporcional en la tasa de embarazo y la edad de la adolescente, con 2,9\% entre mujeres de 15 años hasta 26,4\% en mujeres de 19 años; siendo esta tasa aún mayor en departamentos de la selva y sierra, sobre todo en áreas rurales ${ }^{3}$. El departamento de Ica no es ajena a esta realidad, considerando que su tasa de EA es superior al estimado para todo el Perú, llegando al $11,3 \%$ de adolescentes que ya son madres y $4,7 \%$ para aquellas que están embarazadas de su primer hijo ${ }^{1}$.

La misma tendencia de incremento se observa para el bajo peso al nacer; así, en el año 2015 se registró que el 7\% de los recién nacidos vivos tuvieron una tasa de $7 \%$ con bajo peso ${ }^{4}$. El Perú es uno de los países con mayor tasa de EA y bajo peso al nacer (BPN) en Latinoamérica ${ }^{5}$. Las investigaciones que evidencien asociación entre estas dos entidades epidemiológicas son limitadas en nuestro país; por ejemplo, en la amazonía peruana se ha reportado asociación significativa entre BPN y EA entre 10 a 14 años de edad, en comparación a madres mayores de 19 años ${ }^{6}$; asimismo, a nivel internacional, las evidencias son consistentes y demuestran asociación significativa ${ }^{7,8}$. Por otro lado, existe limitada investigación nacional que evidencie la asociación entre EA e hiperbilirrubinemia neonatal (HN), y tampoco datos de prevalencia para esta última condición, aunque algunos autores señalan que puede oscilar entre un 8 a $11 \%{ }^{9}$; mientras que otros, han encontrado asociación entre EA e $\mathrm{HN}^{10}$. Sin embargo, aún existe una brecha muy grande por cubrir, en relación al estudio de la hiperbilirrubinemia y el BPN asociado a EA; más aún en el departamento de Ica, donde la problemática descrita se presenta con una de las mayores prevalencias en Perú.

Ante las limitadas evidencias científicas generadas en nuestro país, la presente investigación tuvo por objetivo estimar la asociación entre embarazo adolescente e hiperbilirrubinemia con el bajo peso al nacer en una cohorte retrospectiva de gestantes adolescentes evaluadas en un Hospital de Perú, durante los años 2015 y 2016. Esto resulta de vital importancia para la identificación de grupos de riesgo asociados a desenlaces adversos a la salud que a su vez guardan asociación con la morbi-mortalidad neonatal, información que puede utilizarse para priorizar sistemas de vigilancia y monitoreo gineco-obstétrico en este tipo de población.

\section{MÉTODOS}

Se realizó un estudio de cohorte retrospectivo con dos grupos de comparación: gestantes adolescentes menores a 18 años de edad y de 18 años de edad a más.

\section{Población y muestra de estudio}

Estuvo constituido por todos los registros de gestantes adolescentes (de 19 años de edad a menos) atendidas y controladas ( $n=496)$ durante los años 2015 y 2016 en el Hospital Santa María del Socorro de Ica. Las historias clínicas fueron proporcionadas para su revisión por el área de gineco obstetricia del Hospital Santa María del Socorro del departamento de Ica en Perú, una institución estatal de nivel de atención III. Se excluyeron registros con datos faltantes del peso del recién nacido, hiperbilirrubinemia o edad gestacional; asimismo, no se incluyeron recién nacidos fallecidos. El flujograma de seguimiento se muestra a continuación:

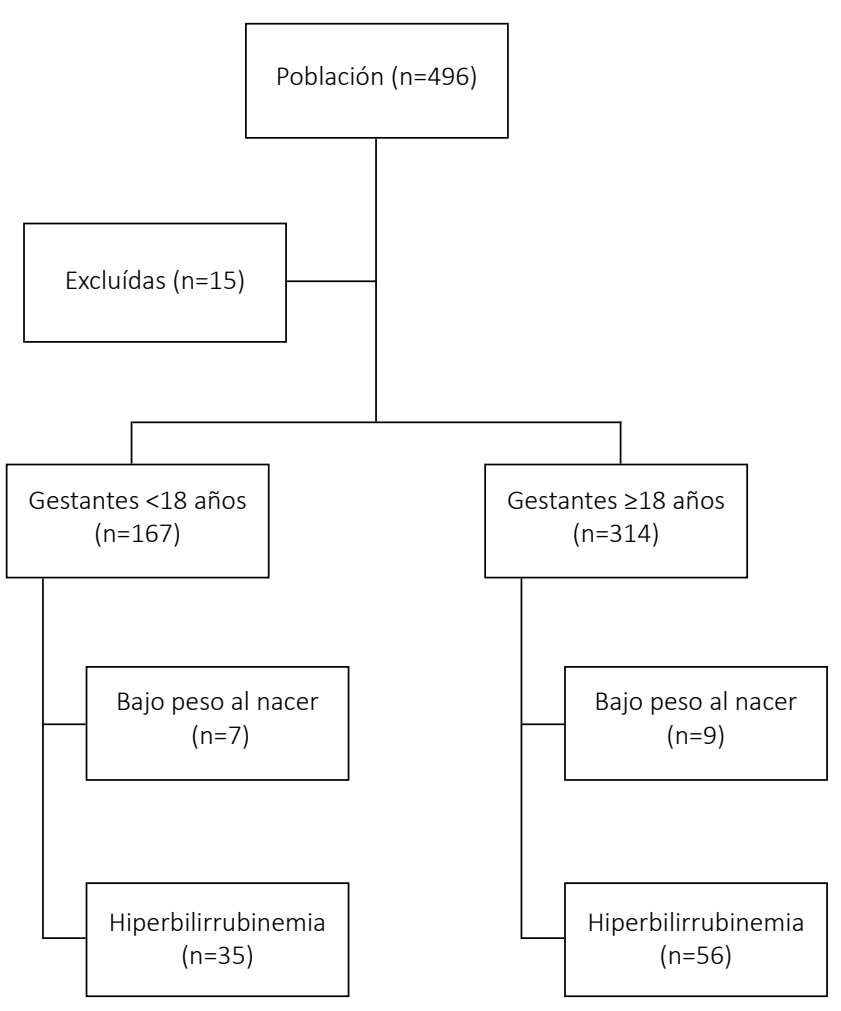


Dado que se trabajó con la población atendida en 2 años, se estimó la potencia en un modelo de regresión de Cox de riesgos logarítmicos ${ }^{11}$ con una variable de respuesta binaria ( $\mathrm{Y}$ : presencia o ausencia de bajo peso al nacer), en una variable independiente ( $X$ : embarazo adolescente), con una desviación estándar de 2,5 años, y cuyo valor obtenido fue de $90,3 \%$ a un nivel de significancia de 0,05, con capacidad para detectar un coeficiente de regresión de 0,7 (equivalente a un Hazard Ratio de 2). El tamaño de muestra fue ajustada en un modelo de regresión de Cox múltiple, con un coeficiente $R^{2}$ de 0,9 con capacidad para explicar el modelo presentado. Finalmente, la potencia fue estimada considerando una proporción de evento de 0,07 (bajo peso al nacer según lo reportado por el Ministerio de Salud de Perú en su Boletín estadístico de nacimiento ${ }^{4}$. El cálculo de potencia fue realizado con el módulo estadístico para estimación de tamaño de muestra PASS (Power Analysis and Sample Size), versión $11^{12}$.

\section{Ficha epidemiológica}

Fue elaborada con el objetivo de recabar información relacionada a las variables de exposición (embarazo adolescente) y desenlace (bajo peso al nacer: recién nacidos con peso menor a 2500 gramos, e hiperbilirrubinemia: recién nacidos con bilirrubina total mayor a 14 $\mathrm{mg} / \mathrm{dL}$ ), las cuales fueron definidas según niveles de edad cronológica de la gestante, peso del recién nacido y concentración de la bilirrubina total, directa e indirecta (medidas durante las 24 horas post parto, pero también al tercer y quinto día como parte del monitoreo neonatal). También se obtuvo información relacionada a potenciales variables confusoras como edad gestacional, tipo de parto, primípara, diabetes gestacional (definida como toda gestante con un nivel de glicemia mayor o igual a $92 \mathrm{mg} / \mathrm{dL}$ según criterios de la OMS), hipertensión arterial, obesidad (definida a toda gestante con un IMC mayor a 29,99 $\mathrm{Kg} / \mathrm{m}^{2}$ ), pre-eclampsia, anemia materna (definida como toda gestante con un nivel de hemoglobina menor a $11 \mathrm{~g} / \mathrm{dL}$ para aquellas que fueron evaluadas en el primer y tercer trimestre), e infección urinaria.

\section{Análisis estadístico}

Se realizó un análisis descriptivo: para las variables numéricas se calculó medidas de tendencia central y dispersión (media y desviación estándar). Las variables categóricas se presentan en frecuencias absolutas y relativas. La presencia o ausencia de BPN e hiperbilirrubinemia neonatal fueron comparadas según variables categóricas mediante la prueba chi cuadrado de Pearson; mientras que las medianas de las variables numéricas, mediante la prueba de prueba no paramétrica de Mann-Withney, previa evaluación de supuestos de normalidad e independencia de mediciones para esta última. En ambos casos, se consideró como diferencia significativa un valor de probabilidad menor a 0,05. Considerando que el diseño corresponde a un estudio de cohortes, y la variable de interés fue de tiempo-evento (edad gestacionalBPN o hiperbilirrubinemia), y con escala de medición dicotómica, la asociación fue evaluada mediante el valor del $\mathrm{Ha}$ zard Ratio (HR, obtenido del cálculo exponencial del coeficiente de regresión $\beta$ ) calculado en un modelo de regresión de Cox multiple ajustado por potenciales variables confusoras seleccionadas en base a un criterio epidemiológico. Además, se representó el intervalo de confianza al 95\% y valor de probabilidad para evaluar asociación significativa, siendo esta con valores menores a 0,05. Todos los cálculos fueron realizados con el software Stata Corporation versión 14.0.

Tabla 1. Características de las gestantes adolescentes atendidas en el Hospital Santa María del Socorro de Ica, Perú, 2015-2016.

\begin{tabular}{lll} 
Variable de estudio & \multicolumn{1}{c}{$\begin{array}{c}\text { Adolescente gestante }(\mathbf{x} \pm \mathbf{D E}) \\
\mathbf{2} \text { (18 años }\end{array}$} \\
\hline Edad, años & $16,2 \pm 0,9$ & $18,5 \pm 0,5$ \\
\hline Edad gestacional, semanas & $38,6 \pm 1,5$ & $38,8 \pm 1,4$ \\
\hline $\mathrm{IMC}, \mathrm{kg} / \mathrm{m}^{2}$ & $27,9 \pm 4,2$ & $28,5 \pm 4,1$ \\
\hline Glicemia materna, $\mathrm{mg} / \mathrm{dL}$ & $82,9 \pm 8,9$ & $82,5 \pm 9,8$ \\
\hline Hemoglobina materna, $\mathrm{g} / \mathrm{dL}$ & $11,2 \pm 1,3$ & $11,1 \pm 1,2$ \\
\hline Peso del recién nacido $(\mathrm{RN}), \mathrm{g}$ & $64,8 \pm 10,5$ & $66,7 \pm 10,4$ \\
\hline Bilirrubina total del $\mathrm{RN}, \mathrm{mg} / \mathrm{dL}$ & $14,1 \pm 3,9$ & $14,3 \pm 3,9$ \\
\hline Bilirrubina indirecta del $\mathrm{RN}, \mathrm{mg} / \mathrm{dL}$ & $13,2 \pm 4,2$ & $13,7 \pm 3,7$ \\
\hline
\end{tabular}

$x \pm D E:$ media y desviación estándar

\section{Consideraciones éticas}

La investigación fue presentada, aprobada y autorizada para su ejecución por la Dirección General del Hospital Santa María del Socorro de Ica, Perú, así como por la Universidad Alas Peruanas de Ica, Perú. Se mantuvieron en confidencialidad los datos de las historias clínicas evaluadas.

\section{RESULTADOS}

Se evaluaron 496 registros de gestantes adolescentes, de los cuales se excluyeron 15 por incumplimiento de criterios. Los resultados muestran que de las 481 gestantes adolescentes evaluadas, el 5\% fueron menores de 15 años de edad y presentaron menos de 16 semanas de edad gestacional. Asimismo, el 5\% de las evaluadas presentaron glicemias menores a $70 \mathrm{mg} / \mathrm{dL}$ (correspondiente a gestantes hipoglicémicas), y hemoglobina menor a $9 \mathrm{~g} / \mathrm{dL}$ (correspondientes a anemia severa según criterios definidos por OMS). Por otra parte, el 5\% de las evaluadas al inicio de su embarazo, presentaron obesidad grado II, mientras que el $1 \%$ obesidad grado III. También se encontró que el BPN e hiperbilirrubinemia neonatal presentaron frecuencias de 3,3\% y $18,9 \%$, respectivamente. Estos datos se muestran en la tabla 1.

Las variables que presentaron diferencias significativas entre los recién nacidos con y sin bajo peso fueron la edad gestacional y el tipo de parto. Se encontró una menor mediana de edad gestacional 
(36 semanas) en las madres de recién nacidos con bajo peso, comparado a la mediana de edad gestacional (39 semanas) en madres de recién nacidos sin bajo peso. Del mismo modo, las gestantes que fueron atendidas por parto normal tuvieron una proporción de BPN de 1,9 $\%$, comparado a las gestantes atendidas por cesárea con recién nacidos con bajo peso de 1,5\%. En el caso de las gestantes adolescentes, no se encontró diferencias entre grupos. En el caso de la hiperbilirrubinemia, no se encontraron variables que presenten diferencias significativas según la presencia o ausencia de hiperbilirrubinemia en recién nacidos, tal como se muestra en la tabla 2.

Para estimar la asociación entre BPN y $E A$, se realizó el análisis de regresión de Cox con cálculo de Hazard Ratio (HR) como medida de asociación y sus intervalos de confianza para identificar si hubo asociación significativa. Se evidenció una asociación significativa entre EA y BPN. Las gestantes adolescentes menores de 18 años de edad tuvieron casi 8 veces más riesgo (nivel de asociación) de tener un recién nacido con bajo peso, en com- paración a las gestantes adolescentes de 18 años de edad a más, además presentando un valor significativo $(p<0,05)$ y con un intervalo de confianza al 95\% de 1,1 a 60,4, según se muestra en la tabla 3 .

Para el caso de la asociación de hiperbilirrubinemia en recién nacidos y EA, no se encontró asociación significativa entre ambas variables, ni incluso con los valores de HR calculados a través de la regresión bivariada. Tabla 4.

\section{DISCUSIÓN}

EI BPN es uno de los principales factores determinantes de la supervivencia perinatal, la morbilidad y mortalidad infantil, así como el riesgo de discapacidades y enfermedades del desarrollo en vidas futuras. La OMS calcula que cada año nacen alrededor de 30 millones de bebés con bajo peso al nacer (el $23,4 \%$ de todos los nacimientos) y que a menudo se enfrentan a eventos adversos de salud a corto y largo plazo. Mientras que la prevalencia mundial de BPN ha disminuido ligeramente, la tasa en muchos países en desarrollo sigue siendo bastante alta ${ }^{13}$. En nuestro país, el BPN entre las madres adolescentes es un problema de salud pública ${ }^{1}$.

Los resultados presentados evidencian un serio problema materno infantil, considerando que la prevalencia de embarazo adolescente fue de $34,7 \%$ en las gestantes atendidas entre los años 2015 y 2016 en el Hospital Santa María del Socorro de Ica. Sin embargo, la prevalencia de BPN fue de $3,3 \%$, cifras similares a lo reportado en otros estudios nacionales. Por ejemplo, Herrera y col., reportaron en el año 2015 en Lima, una mediana de peso en recién nacido de $3350 \mathrm{~g}$ (la nuestra tuvo un promedio de $3316,5 \mathrm{~g}$ ), y la prevalencia fue del $2,4 \%$ de bajo peso al nacer ${ }^{14}$.

Por otra parte, el contraste de hipótesis por análisis bivariado evidenció que la edad gestacional era menor significativamente $(p=0,000)$ en las madres con recién nacidos de bajo peso (mediana de 36 semanas), comparados a aquellas madres con recién nacidos sin bajo peso (mediana de 39 semanas). Esto evidencia

Tabla 2. Análisis bivariado entre BPN, hiperbilirrubinemia y variables independientes de gestantes adolescentes atendidas en el Hospital Santa María del Socorro de Ica, Perú, 2015-2016.

\begin{tabular}{|c|c|c|c|c|c|c|}
\hline \multirow{2}{*}{ Variable de estudio } & \multicolumn{2}{|c|}{ Bajo peso al nacer } & \multirow{2}{*}{ p valor } & \multicolumn{2}{|c|}{ Hiperbilirrubinemia } & \multirow{2}{*}{ p valor } \\
\hline & No & Si & & No & Si & \\
\hline Edad gestacional & $39(2)$ & $36(2,5)$ & $*^{*} 0,000$ & $39(2)$ & $39(2)$ & $* 0,988$ \\
\hline $\mathrm{IMC}, \mathrm{kg} / \mathrm{m}^{2}$ & $27,8(5,4)$ & $26,9(3,9)$ & $* 0,590$ & $27,8(5,1)$ & $27,1(6.4)$ & $* 0,227$ \\
\hline Glicemia materna, mg/dL & $81(12)$ & $81(15)$ & $* 0,976$ & $81(12)$ & $80(14)$ & $* 0,143$ \\
\hline Hemoglobina materna, g/dL & $11,3(1,5)$ & $10,8(1,8)$ & *0,182 & $11,3(1,5)$ & $11,2(2)$ & $* 0,810$ \\
\hline Tipo de parto (\%) & & & $* * 0,014$ & & & $* * 0,553$ \\
\hline Normal & 78,4 & 1,9 & & 65,5 & 14,8 & \\
\hline Cesárea & 18,3 & 1,4 & & 15,6 & 4,2 & \\
\hline Primípara (\%) & & & $* * 0,704$ & & & $* * 1,000$ \\
\hline No & 14,8 & 0,6 & & 12,5 & 2,9 & \\
\hline $\mathrm{Si}$ & 81,9 & 2,7 & & 68,6 & 16,0 & \\
\hline Infección urinaria(\%) & & & $* * 0,416$ & & & $* * 0,205$ \\
\hline No & 27,2 & 1,3 & & 24,1 & 4,4 & \\
\hline $\mathrm{Si}$ & 69,4 & 2,1 & & 57,0 & 14,5 & \\
\hline HTA (\%) & & & $* * 0,364$ & & & ${ }^{* *} 0,418$ \\
\hline No & 90,2 & 2,8 & & 75,9 & 17,3 & \\
\hline $\mathrm{Si}$ & 6,4 & 0,4 & & 5,2 & 1,6 & \\
\hline Embarazo adolescente (\%) & & & $* * 0,440$ & & & $* * 0,405$ \\
\hline$\geq 18$ años & 33,3 & 1,5 & & 27,4 & 7,3 & \\
\hline$<18$ años & 63,4 & 1,9 & & 53,6 & 11,7 & \\
\hline
\end{tabular}

*Mann-Withney, **Prueba chi-cuadrado 
Tabla 3. Análisis multivariado entre bajo peso al nacer, embarazo adolescente y variables independientes, de gestantes adolescentes atendidas en el Hospital Santa María del Socorro de Ica, Perú, 2015-2016.

\begin{tabular}{|c|c|c|c|c|}
\hline \multirow{2}{*}{ Bajo peso al nacer } & \multicolumn{2}{|c|}{ Modelo bivariado } & \multicolumn{2}{|c|}{ Modelo multivariado* } \\
\hline & HR & IC 95 & HR & IC 95 \\
\hline $\mathrm{IMC}, \mathrm{kg} / \mathrm{m}^{2}$ & 1,02 & $0,86-1,21$ & $\ldots$ & $\ldots$ \\
\hline Glicemia materna, mg/dL & 1,01 & $0,95-1,09$ & 1,08 & $0,98-1,18$ \\
\hline Hemoglobina materna, g/dL & 0,76 & $0,53-1,10$ & 0,57 & $0,31-1,07$ \\
\hline \multicolumn{5}{|l|}{ Tipo de parto (\%) } \\
\hline Normal & \multicolumn{2}{|c|}{ Ref. } & \multicolumn{2}{|c|}{ Ref. } \\
\hline Cesárea & 3,29 & $1,05-10,24$ & 1,55 & $0,27-8,83$ \\
\hline \multicolumn{5}{|l|}{ Primípara (\%) } \\
\hline No & \multicolumn{2}{|c|}{ Ref. } & \multicolumn{2}{|c|}{ Ref. } \\
\hline $\mathrm{Si}$ & 0,92 & $0,25-3,37$ & $\ldots$ & $\ldots$ \\
\hline \multicolumn{5}{|l|}{ Infección urinaria (\%) } \\
\hline No & \multicolumn{2}{|c|}{ Ref. } & \multicolumn{2}{|c|}{ Ref. } \\
\hline $\mathrm{Si}$ & 0,29 & $0,09-0,88$ & 0,02 & $0,00-0,26$ \\
\hline \multicolumn{5}{|l|}{ Hipertensión arterial (\%) } \\
\hline No & \multicolumn{2}{|c|}{ Ref. } & \multicolumn{2}{|c|}{ Ref. } \\
\hline $\mathrm{Si}$ & 1,27 & $0,28-5,79$ & $\ldots$ & $\ldots$ \\
\hline \multicolumn{5}{|l|}{ Embarazo adolescente (\%) } \\
\hline$\geq 18$ años & \multicolumn{2}{|c|}{ Ref. } & \multicolumn{2}{|c|}{ Ref. } \\
\hline$<18$ años & 0,68 & $0,23-1,95$ & 7,95 & $1,05-60,40$ \\
\hline
\end{tabular}

* Regresión de Cox ajustado por método de Breslow para empates. Ajustado por glicemia y hemoglobina materna, tipo de parto, e infección urinaria. HR: Hazard Ratio, IC95: Intervalo de confianza al 95\%, Ref.: Grupo de referencia o comparación

que el parto pre término puede repre- gos de edad, pero donde la diferencia de sentar un potencial factor de riesgo para años no es tan grande, es probable que el bajo peso al nacer. Asimismo, dado la diferencia sea mucho mayor con resque los grupos de comparación son entre pecto a gestantes adultas. Esto fue algo madres adolescentes con distintos ran- que Gonzáles y col. reportaron en un es- tudio realizado en Trujillo en el año 2014, donde encontró que ser madre menor de 19 años es un factor de riesgo para el bajo peso en recién nacidos a término, en comparación con las madres mayores de

Tabla 4. Análisis multivariado entre hiperbilirrubinemia, embarazo adolescente y variables independientes, de gestantes adolescentes atendidas en el Hospital Santa María del Socorro de Ica, Perú, 2015-2016.

\begin{tabular}{|c|c|c|c|c|}
\hline \multirow{2}{*}{ Hiperbilirrubinemia } & \multicolumn{2}{|c|}{ Modelo bivariado } & \multicolumn{2}{|c|}{ Modelo multivariado* } \\
\hline & HR & IC 95 & HR & IC 95 \\
\hline $\mathrm{IMC}, \mathrm{kg} / \mathrm{m}^{2}$ & 0,99 & $0,96-1,04$ & $\ldots$ & $\ldots$ \\
\hline Glicemia materna, mg/dL & 1,00 & $0,98-1,03$ & 1,00 & $0,98-1,03$ \\
\hline Hemoglobina materna, g/dL & 1,02 & $0,88-1,20$ & 1,03 & $0,88-1,21$ \\
\hline \multicolumn{5}{|l|}{ Tipo de parto (\%) } \\
\hline Normal & \multicolumn{2}{|c|}{ Ref. } & \multicolumn{2}{|c|}{ Ref. } \\
\hline Cesárea & 0,86 & $0,52-1,41$ & 0,86 & $0,51-1,47$ \\
\hline \multicolumn{5}{|l|}{ Primípara (\%) } \\
\hline No & \multicolumn{2}{|c|}{ Ref. } & \multicolumn{2}{|c|}{ Ref. } \\
\hline $\mathrm{Si}$ & 0,90 & $0,51-1,59$ & $\ldots$ & $\ldots$ \\
\hline \multicolumn{5}{|l|}{ Infección urinaria (\%) } \\
\hline No & \multicolumn{2}{|c|}{ Ref. } & \multicolumn{2}{|c|}{ Ref. } \\
\hline $\mathrm{Si}$ & 0,38 & $0,86-2,42$ & 1,48 & $0,88-2,50$ \\
\hline \multicolumn{5}{|l|}{ Hipertensión arterial (\%) } \\
\hline No & \multicolumn{2}{|c|}{ Ref. } & \multicolumn{2}{|c|}{ Ref. } \\
\hline $\mathrm{Si}$ & 1,51 & $0,73-3,13$ & $\ldots$ & $\ldots$ \\
\hline \multicolumn{5}{|l|}{ Embarazo adolescente (\%) } \\
\hline$\geq 18$ años & \multicolumn{2}{|c|}{ Ref. } & \multicolumn{2}{|c|}{ Ref. } \\
\hline$<18$ años & 1,18 & $0,77-1,81$ & 1,22 & $0,79-1,88$ \\
\hline
\end{tabular}

* Regresión de Cox ajustado por método de Breslow para empates. Ajustado por glicemia y hemoglobina materna, tipo de parto e infección urinaria.

HR: Hazard Ratio, IC95: Intervalo de confianza al 95\%, Ref.: Grupo de referencia o comparación 
35 años que no lo son ${ }^{15}$. Asimismo, Ticona y Huanco realizaron estudios en Tacna en los años 2000 y 2012, donde concluyeron que la edad materna, escolaridad y paridad influyen significativamente en el peso del RN a término de hospitales del Ministerio de Salud del Perú ${ }^{16}$; además, que los recién nacidos de madres adolescentes presentan significativamente un mayor riesgo de prematuridad (RR: 1,63 ) y bajo peso al nacer (RR: 1,36), que los recién nacidos de madres adultas. Todos estos factores determinan un incremento en la mortalidad neonatal precoz, la cual resulta siendo el doble de la encontrada en los hijos de madres adultas ${ }^{17}$. Estudios internacionales evidencian la misma problemática reportada en nuestro estudio. Por ejemplo, Naz U. en Pakistan, concluyó que las adolescentes primíparas tuvieron un riesgo significativamente mayor de resultados adversos del embarazo, como el parto prematuro, recién nacidos de bajo peso, y anemia, en comparación con las primíparas adultas ${ }^{18}$.

El análisis de regresión de Cox realizado, evidenció que el embarazo adolescente en menores de 18 años de edad generó un riesgo de casi 8 veces (HR: 7,95) a tener un recién nacido con bajo peso, en comparación a aquellas de 18 años de edad a más. Por tanto, la edad materna se relaciona directamente con el peso del recién nacido. Este hallazgo también ha sido reportado por Bedoya en Puno durante el año 2017, quien evidenció factores de riesgo maternos asociados al BPN como ser adolescente (OR:1,12), bajo peso materno (OR:1,12), deficiente ganancia de peso durante el embarazo (OR: 15,2), bajo ingreso familiar (OR: 11,87), grado de instrucción deficiente (OR: 7,66), hábito de fumar (OR: 5,37), deficiente número de controles prenatales (OR: 5,25$)$ y anemia materna durante el embarazo (OR: 3.83), considerados como factores de riesgo ${ }^{19}$. Investigadores extranjeros también han evidenciado que el embarazo antes de los 20 años de edad representa un factor de riesgo (OR: $1,71)$ importante para el $B P N^{20}$. Y recientemente, se ha evidenciado que la edad materna es una variable que guarda estrecha relación con el $\mathrm{BPN}^{21}$. En ese aspecto, nuestros hallazgos son coherentes con las evidencias científicas reportadas a la fecha; y también son importantes, considerando que el peso en el recién nacido, es un indicador relevante que se considera dentro de las guías relacionadas a los programas de inmunización neonatal dependiendo del peso 22 .

Nuestros resultados no evidenciaron asociación entre bajo peso al nacer y tipo de parto, situación que si ha sido evidenciada por otros investigadores, como Santos N. et al., quienes encontraron en Brasil durante el 2014, que en el embarazo adolescente menor de 16 años, el parto por cesárea tuvo asociación significativa con recién nacidos de bajo peso (OR 1,34) ${ }^{23}$. Sin embargo, es importante mencionar que en nuestro estudio se presentaron algunas limitaciones debido al registro incompleto de historias clínicas en relación a potenciales factores de riesgo asociados a bajo peso al nacer, tales como el tabaquismo, el nivel socioeconómico y académico, e incluso la etnicidad. Por ejemplo, Guimarães et al., evidenciaron en Brasil en el año 2013, que la adolescencia fue un factor de riesgo para el bajo peso al nacer; de igual manera, el tabaquismo durante el embarazo y la falta de atención prenatal se consideraron factores de riesgo independientes para el bajo peso al nacer ${ }^{8}$. Por otra parte, Harville et al. encontraron en Estados Unidos en el año 2012, que en las adolescentes no negras, el índice de masa corporal más bajo estuvo asociado al bajo peso al nacer, mientras que estar soltera se asoció con una menor edad gestacional ${ }^{24}$. Asimismo, Dos Santos et al., en el año 2008, concluyeron que el embarazo en la adolescencia se asoció a la baja escolaridad (OR: 1,95) y bajo peso al nacer (OR: 1,5) ${ }^{25}$.

Otro problema identificado en los recién nacidos y que representa un alto riesgo para la vida y salud del neonato, es la presencia de hiperbilirrubinemia, evidenciada clínicamente como ictericia neonatal. La ictericia es un evento común que se observa en el $60 \%$ del neonato a término y el $80 \%$ de los prematuros en el momento del nacimiento, y con frecuencia es una hiperbilirrubinemia benigna con una bilirrubina total mayor al percentil del 95. La presente investigación reportó hiperbilirrubinemia en el $18,9 \%$ de los recién nacidos evaluados, considerando un nivel de bilirrubina total por encima del percentil 95, que para nuestro caso fue superior a $14 \mathrm{mg} / \mathrm{dL}$ durante las primeras 24 horas post parto, el cual ya es considerado como una hiperbilirrubinemia patológica, que requiere monitoreo y tratamiento específico según las categorías de ictericia definidas según los criterios de Kramer $^{26}$, el cual además presenta una alta correlación con los niveles de bilirrubina total ${ }^{27}$. En Perú, no se ha reportado prevalencias de hiperbilirrubinemia asociada a embarazo adolescente; sin embargo, en otros países, existen publicaciones al respecto. Así, Khadije et al., concluyeron que la hiperbilirrubinemia indirecta neonatal severa es una condición muy prevalente y su prevención depende mucho de los antecedentes étnicos y culturales de las madres, incluso más que la educación escolar $^{28}$. La hiperbilirrubinemia es una condición muy grave si es que no recibe tratamiento oportuno, incluso con alto riesgo de desarrollar disfunción neurológica permanente inducida por la bilirrubina. La encefalopatía aguda por bilirrubina clásicamente se denomina Kernicterus, la cual significa secuela crónica y permanente de la disfunción neurológica por bilirrubina. La intervención adecuada, como fototerapia y exsanguíneo transfusión, se usa en todos los bebés con hiperbilirrubinemia grave. Incluso si estos bebés reciben un tratamiento adecuado, no se puede prevenir la secuela neurológica a largo plazo. La identificación de la causa de la hiperbilirrubinemia neonatal es útil para determinar si las intervenciones terapéuticas pueden prevenir la hiperbilirrubinemia grave. En nuestro estudio, no hubo reportes de presencia de Kernicterus; sin embargo, otros estudios reportan tasas bajas para esta patología. Por ejemplo, Choudhury et al. reportaron Kernicterus en nueve $(2,1 \%)$ recién nacidos, cuatro de los cuales sobrevivieron con secuelas neurológicas. Además, indicaron que la incompatibilidad $\mathrm{ABO}$ es dos veces más común que la incompatibilidad Rh y la mayoría de los pacientes con Kernicterus murieron en fase aguda ${ }^{29}$. No obstante, según lo reportado en la literatura, no es concluyente la asociación entre embarazo adolescente e hiperbilirrubinemia, debido a los escasos estudios sobre el tema. Nuestros resultados 
no evidenciaron asociación significativa entre ambas variables, y tampoco hubo alguna variable asociada a la presencia de hiperbilirrubinemia neonatal.

Las limitaciones del estudio debido al diseño retrospectivo, surgen ante la revisión de historias clínicas de las gestantes, la cual estuvo limitada a las variables presentadas en el estudio; sin embargo, no se pudo obtener información como el tabaquismo, estado nutricional, socioeconómico y nivel académico, las cuales han sido reportadas como factores asociados al BPN. Tampoco se incluyó casos con pre-eclampsia, considerando que esta fue catalogada únicamente según la presencia de hipertensión, y no de acuerdo a las recomendaciones establecidas por la Organización Mundial de la Salud y Ministerio de Salud del Perú, quienes la definen esta condición, además, con la presencia de proteinuria mayor a $300 \mathrm{mg}$ en orina de 24 horas.

Finalmente, podemos mencionar que nuestros resultados evidencian que el bajo peso al nacer se encuentra asociado al embarazo adolescente, sobre todo en aquellas gestantes que son menores a los 18 años de edad, y que por tanto deberían recibir controles perinatales y educación continua para ser informadas sobre los cuidados a tener en consideración durante el embarazo. En conclusión, según nuestros hallazgos, el embarazo adolescente en menores de 18 años de edad se encuentra asociado significativamente al BPN, más no a la hiperbilirrubinemia neonatal.

\section{REFERENCIAS BIBLIOGRÁFICAS}

1. Instituto Nacional de Estadística e Informática. Las adolescentes y su comportamiento reproductivo 2013. Lima, Perú: Instituto Nacional de Estadística e Informática. 2013.

2. Instituto Nacional de Estadística e Informática. Perú: Maternidad en la adolescencia, 2015. Lima, Perú: Instituto Nacional de Estadistica e Informática. 2015.
3. Instituto Nacional de Estadística e Informática. Perú: Encuesta Demográfica y de Salud Familiar-ENDES 2017. Lima, Perú: Instituto Nacional de Estadistica e Informática. 2018.

4. Ministerio de Salud del Perú. Boletin estadistico de nacimientos Perú: 2015. Lima, Perú: Ministerio de Salud. 2016.

5. Fondo de las Naciones Unidas para la Infancia, UNICEF. Niñas y adolescentes en América Latina y el Caribe. Santiago de Chile: Fondo de las Naciones Unidas para la Infancia. 2016.

6. Ryan JA. Adolescent pregnancy and low birth weight in the Peruvian Amazon (Thesis submitted to McGill University in partial fulfillment of the requirements of the degree of Master of Science). Montreal: McGill University; 2009.

7. dos Santos GH, Martins Mda G, Sousa Mda S. Teenage pregnancy and factors associated with low birth weight. Rev. Bras. Ginecol. Obstet. 2008;30(5):224-31. DOI: http://dx.doi.org/10.1590/ S0100-72032008000500004

8. Guimaraes AM, Bettiol H, Souza L, Gurgel RQ, Almeida ML, Ribeiro ER, et al. Is adolescent pregnancy a risk factor for low birth weight?. Revista de saude publica. 2013;47(1):11-9. DOI: http://dx.doi. org/10.1590/S0034-89102013000100003

9. Ullah S, Rahman K, Hedayati M. Hyperbilirubinemia in Neonates: Types, Causes, Clinical Examinations, Preventive Measures and Treatments: A Narrative Review Article. Iranian Journal of Public Health. 2016;45(5):558-68.

10. Kumar A, Singh T, Basu S, Pandey S, Bhargava $\mathrm{V}$. Outcome of teenage pregnancy. Indian journal of pediatrics. 2007;74(10):927-31. DOI: 10.1007/ s12098-007-0171-2

11. Hsieh FY, Lavori PW. Sample-size calculations for the Cox proportional hazards regression model with nonbinary covariates. Controlled clinical trials. 2000;21(6):552-60.

12. Hintze J. Power Analysis and Sample Size System. User’s Guide I. Utah, USA: NCSS Statistical Software. 2011.

13. Organización Panamericana de la Salud [Internet]. América Latina y el Caribe tienen la segunda tasa más alta de embarazo adolescente en el mundo [Fecha de acceso: 12 de febreo 2019]. Disponible en: https://tinyurl.com/yccdmk57

14. Herrera Risco J. Relación entre la ganancia ponderal de la gestante y el peso del recién nacido en el Centro Materno Infantil "César López Silva" del Distrito de Villa El Salvador, Lima, Perú 2011 (Tesis para obtener el grado académico de magister en nutrición). Lima: Universidad Nacional Mayor de San Marcos; 2015

15. Gonzales J. Edad materna como factor de riesgo para bajo peso del recién nacido a término (Tesis para obtener el título de médico cirujano). Trujillo, Perú: Universidad Privada Antenor Orrego; 2014.

16. Ticona M, Huanco D. Caracteristicas del peso al nacer en el Perú: Incidencia, factores de riesgo y morbimortalidad. Tacna, Perú: Concytec. 2012.
17. Ticona M. Riesgos en el recién nacido de madre adolescente en el Hospital Hipólito Unanue de Tacna. Diagnóstico. 2000;39(1):52-9.

18. Naz U. Comparison of obstetric outcome in terms of the risk of low birth weight, preterm delivery, cesarean section rate and anemia in primigravid adolescents and older primigravida. Journal of the College of Physicians and Surgeons Pakistan. 2014;24(2):131-4. DOI: 02.2014/jcpsp.131134

19. Bedoya A. Factores maternos que influyen en el bajo peso del recién nacido en el Hospital Regional de Ayacucho, periodo enero-junio 2016 (Tesis para optar el título profesional de médico cirujano). Puno, Perú: Universidad Nacional del Altiplano; 2017.

20. Gebregzabiherher Y, Haftu A, Weldemariam S, Gebrehiwet $\mathrm{H}$. The Prevalence and Risk Factors for Low Birth Weight among Term Newborns in Adwa General Hospital, Northern Ethiopia. Obstet Gynecol Int. 2017;2017:2149156. DOI: 10.1155/2017/2149156

21. Tshotetsi L, Dzikiti L, Hajison P, Feresu S. Maternal factors contributing to low birth weight deliveries in Tshwane District, South Africa. PloS one. 2019;14(3):e0213058-e. DOI: 10.1371/journal.pone.0213058

22. Cutland CL, Lackritz EM, Mallett-Moore T, Bardaji A, Chandrasekaran R, Lahariya $\mathrm{C}$, et al. Low birth weight: Case definition \& guidelines for data collection, analysis, and presentation of maternal immunization safety data. Vaccine. 2017;35(48 Pt A):6492-500. DOI: 10.1016/j.vaccine.2017.01.049

23. Santos NL, Costa MC, Amaral MT, Vieira GO Bacelar EB, de Almeida $\mathrm{AH}$. Teenage pregnancy: analysis of risk factors for low birth weight, prematurity and cesarean delivery. Ciencia \& saude coletiva. 2014;19(3):719-26. DOI: http://dx.doi. org/10.1590/1413-81232014193.18352013

24. Harville EW, Madkour AS, Xie Y. Predictors of Birth Weight and Gestational Age Among Adolescents. American Journal of Epidemiology. 2012;176(Suppl 7):S150-S63. DOI: 10.1093/aje/kws231

25. Santos GHNd, Martins MdG, Sousa MdS. Gravidez na adolescência e fatores associados com baixo peso ao nascer. Rev. Bras. Ginecol. Obstet. 2008;30(5):224-31. DOI: http://dx.doi.org/10.1590/ S0100-72032008000500004

26. Acosta-Torres SM, Torres-Espina MT, Colina-Araujo JA, Colina-Chourio JA. Usefullness of the Kramer's index in the diagnosis of hyperbilirubinemia of the newborn. Invest Clin. 2012;53(2):148-56.

27. Aprillia Z, Gayatri D, Waluyanti FT. Sensitivity, Specificity, and Accuracy of Kramer Examination of Neonatal Jaundice: Comparison with Total Bilirubin Serum. Comprehensive child and adolescent nursing. 2017;40(sup1):88-94. DOI: 10.1080/24694193.2017.1386975

28. Najib KS, Saki F, Hemmati F, Inaloo S. Incidence, RiskFactors and Causes of Severe Neonatal Hyperbilirubinemia in the South of Iran (Fars Province). Iran Red Crescent Med J. 2013;15(3):260-3. DOI: 10.5812/ircmj.3337

29. Rasul CH, Hasan MA, Yasmin F. Outcome of Neonatal Hyperbilirubinemia in a Tertiary Care Hospital in Bangladesh. Malays J Med Sci. 2010;17(2):40-4. 